\title{
To determining the mass of a gravitational body with micro-arcsecond astrometric data
}

\author{
H. Cheng, Z. H. Tang and C. Huang \\ Shanghai Astronomical Observatory, Chinese Academy of Science, \\ Nandan Road 200030, Shanghai, China \\ email: chz@shao.ac.cn zhtang@shao.ac.cn
}

\begin{abstract}
A beam of light from a background source to an observer is deflected by a gravitational body when travelling through the space in its vicinity. The deflection changes with configuration of the background source, the gravitational body and the observer, and the amount of deflection is dependent on the gravitational body's mass. It is anticipated that its mass could be determined if the shift can be measured by future precise astrometric projects such as Gaia or SIM-PlanetQuest.
\end{abstract}

Keywords. stars: fundamental parameters (masses), stars: kinematics, gravitation

\section{Introduction}

Determining a gravitational body's mass with its parallax and proper motion data is a method that could be used in the near future, since Gaia or SIM-PlanetQuest would provide micro-arcsecond data from which we can detect tiny shifts in the optical positions of stable background sources, the radiation of which is deflected by nearby gravitational bodies.

New methods for determining the masses of stars by micro-lensing events were developed nearly twenty years ago. The time scale of event and the change in luminosity of the farther star could be used to determine the mass of the nearer star (Gould et al. 1993). In addition, the radius and shape of Einstein rings could be used to determine the deflector's mass (Jiang et al. 2004).

These methods are based on the same principle that relies on the gravitational deflection of light. Although such a deflection always exists, it couldn't be tested until the configuration of the source, deflector and observer changes. In Hosokawa (1993), the optical position of the background source changes when the observer moves (annual parallax), as the light path from the source to the observer changes. Then an expression for the deflector's mass can be obtained. However, this method is only applicable to some nearby stars with large masses. A method suggested by Paczynski (1995) determines the mass of a star by the deflection of light caused by the proper motion of a star. This method is based on the idea similar to Hosokawa's. When compared to parallax, proper motion often causes bigger shifts of deflection per year, and the effects could be added up if the observations last several years, so that the masses of more stars could be obtained.

\section{Calculating the mass with all factors considered}

In theoretical considerations, the shifts caused by both parallax and proper motion should be jointly considered, especially for some nearby stars.

A star with a mass of $1 M_{\odot}$, which is $1 \mathrm{kpc}$ away from the observer and 8 arcsec away from the background source in celestial background, would cause a $1 \mu a s$ shift. 
Here we suppose that the background source is affected by a single star, since even for Gaia normally there will be less than 1 star in $10 \times 10 \mathrm{as}^{2}$. Projecting the observer $(\mathrm{O})$, the gravitational body (GB) and the background source (BS) to the celestial background, the following plot is obtained.

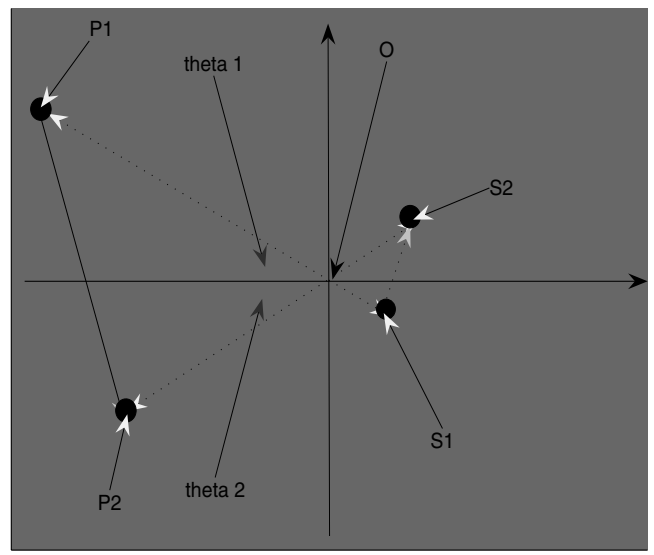

In the above plot, the observer was projected to $\mathrm{O}$, the same point coinciding with the actual position of the background source (BS). $P_{1}, P_{2}$ represent two positions of the gravitational body at time $t_{1}, t_{2}$, while $S_{1}, S_{2}$ represent the optical positions of BS at $t_{1}, t_{2}$, and $\mathrm{D}$ is the distance of the observer from the gravitational body. The final expression of the mass $(\mathrm{M})$ of the gravitational body is given by:

$$
M=\frac{D}{4 G} \frac{P_{1} O * P_{2} O * S_{1} S_{2}}{P_{1} P_{2}}
$$

Here $\mathrm{G}$ is the gravitational constant. To get Fig.1, we should use two overlapping photographs in which shifts caused by other factors had been effaced.

\section{Simulation and Discussion}

In our calculation, the shifts caused by annual parallax can be neglected when the star is more than 100pc away, or is observed in the same season of different years.

If every star in the Hipparcos catalogue would have a mass of $1 M_{\odot}$, there was an ideal background source on the inverse line of its proper motion, the source was 5 arcsec away from any star at the original time $t_{0}$, and the observation lasted for three years. Then the shifts due to gravitational deflection can be calculated. About 8000 Hipparcos stars were found to have the shifts larger than a $1 \mu$ as.

Here sources which have no stars affecting them can be confirmed as motionless. Then the triangular arc method should be applied to confirm positions of sources affected by stars. However, it may be problematic to confirm positions so precisely.

\section{References}

M. Hosokawa, K. Ohnishi, T. Fukushima, \& M. Takeuti, 1993 A\&A. 278, L27-L30.

Paczynski, 1995 astro-ph. /9504099 vl 27 apr.

Andrew Gould, David. P. Bennett, \& David R. Alives, 2004 ApJ. 614:404-411.

Jiang Guangfei, DePoy D. L., Gal-Yam A. et al., 2004 ApJ. 617, 1307J. 